\title{
Comparison of Bayesian and Interval Uncertainty Quantification: Application to the AIRMOD Test Structure
}

\author{
Matteo Broggi*, Matthias Faes ${ }^{\dagger}$, Edoardo Patelli ${ }^{\ddagger}$, Yves Govers ${ }^{\S}$, David Moens ${ }^{\dagger}$, Michael Beer* \\ * Leibniz University Hannover \\ Institute for Risk and Reliability, Callinstrasse 34, 30167 Hannover \\ $\dagger$ KU Leuven \\ Department of Mechanical Engineering, Jan De Nayerlaan 5, 2860 St.-Katelijne-Waver, Belgium \\ $\ddagger$ University of Liverpool \\ Institute for Risk and Uncertainty, Peach Street, L69 7ZF Liverpool \\ $\S$ German Aerospace Center \\ Institute for Aeroelasticity, Bunsenstr. 10,37073 Göttingen
}

\begin{abstract}
This paper concerns the comparison of two inverse methods for the quantification of uncertain model parameters, based on experimentally obtained measurement data of the model's responses. Specifically, Bayesian inference is compared to a novel method for the quantification of multivariate interval uncertainty. The comparison is made by applying both methods to the AIRMOD measurement data set, and comparing their results critically in terms of obtained information and computational expense. Since computational cost of the application of both methods to high-dimensional problems and realistic numerical models can become intractable, an Artificial Neural Network surrogate is used for both methods. The application of this ANN proves to limit the computational cost to a large extent, even taking the generation of the training dataset into account. Concerning the comparison of both methods, it is found that the results of the Bayesian identification provide less overconservative bounds on the uncertainty in the responses of the AIRMOD model.
\end{abstract}

\section{INTRODUCTION}

For the incorporation of non-determinism in numerical design models, two complementary philosophies are commonly applied: the probabilistic and the possibilistic method. Probabilistic methods start from assigning a likelihood to the uncertain parameters of the numerical model under consideration over a defined range, and are aimed at inferring the likelihood of possible realisations of the model responses. Possibilistic approaches such as interval methods on the other hand assign crisp boundaries between which the non-deterministic model parameters are believed to lie, without assigning a likelihood to each value within this range. Consequently, these methods are aimed at inferring the possible worst-case boundaries of the model responses. Elaborate literature exists to date where these concepts are compared both theoretically and practically (see e.g. [1], [2]), even in the context of random processes and interval fields [3]. However, the comparison of probabilistic and interval inverse techniques for the quantification of uncertain model parameters that are not directly measurable, based on a set of measured system responses, is to the knowledge of the authors limited to a single publication by Govers et al. [4]. Therefore, this paper presents the comparison of Bayesian inference, the most commonly applied probabilistic method for performing inverse uncertainty quantification (UQ), with a conceptually new method for the inverse quantification multivariate interval uncertainty that was recently presented by some of the authors [5]. This comparison is specifically made using the AIRMOD test structure [4], [6]. Artificial Neural Networks are trained to limit the computational cost that is associated to both UQ procedures. The obtained results are compared in terms of obtained accuracy, interpretation and computational expense. Section II provides a concise explanation of the AIRMOD test structure and data-set. The results of the Bayesian inference are presented in section III. Section IV presents the interval FE method and the novel quantification method. Section V introduces the neural networks employed in both methods to reduce the overall computational time, and section VI compares the results obtained by both methods. Finally, section VII lists the most important conclusions of the work.

\section{THE AIRMOD TEST STRUCTURE}

The AIRMOD test structure, described in detail in [6], consists of six beam-like components with bolted joint connections, and effectively represent the main vibrational characteristics of an airframe. The structure has been disassembled and reassembled 130 times with identical nominal assembly and tightening characteristics, obtaining a data set comprising 260 vibrational experiments from single point excitation at two locations with the structure supported on soft bungee cords to closely represent free-free boundary conditions. This vibration experiments have been used in the two model updating procedures in order to identify the possibilistic range and probabilistic distribution of the model parameters of a finite element. Through the updating procedure, the finite element model can accurately represent the scatter in the 
eigenfrequencies showed by the experiments. The modes used in updating were the lower modes with the generally simpler shapes shown in 1 . The first four modes are the rigid body modes in yaw, roll, pitch and heave. Modes 5-8 include the first two out-of-plane wing bending modes and the first antisymmetric and the first symmetric wing torsion modes. Mode 10 is the third wing-bending mode. Modes 11 and 12 are the first two in-plane wing bending modes and modes 14, 19 and 20 are local tail-plane modes. This combination of modes ensures that the measured data includes information from all the joints in strained conditions. The modes not included are all the higher-order wing bending modes with more complicated shapes that include also bending and torsion of the tail-plane.

The finite element model used in the parameter updating procedure is realized in NASTRAN and described in [7]. It consisted of $1440 \mathrm{CHEXA}$ and 6 CPENTA elements representing the main aluminium structure, 561 CELAS1 elements that form the elastic connections between the elastic beams at the joints, 55 CMAS1 elements and 18 CONM2 elements that account for additional non-structural masses (such as cables, sensors and bungee connectors) and 3 CROD elements for the bungee chords. Stiffness and mass parameters of the model has been selected for the updating procedure; the stiffness parameters include the stiffness of the supports and joints, and mass parameters are included to represent variability in the position of cable bundles, screws and glue after each reassembly of the structure. The reader might refer to [7] for an insight behind the choice of these parameters.

\section{BAYESIAN INFERENCE}

Bayesian model updating, making use of the well-known Bayes rule, came to the attention of the engineering research community mainly through the pioneering work of Beck and Katafygiotis [8], [9] in the late 1990s - a detailed up-to-date exposition is given by Yuen [10]. The most serious obstacle to practical engineering application is the excessive levels of computer resource needed, but considerable progress has been made in addressing it. The Bayesian updating starts by assigning a prior probability distribution $p(\boldsymbol{\Theta} \mid \mathcal{M})$, conditioned upon a chosen mathematical model $\mathcal{M}$, to a set of uncertain updating parameters $\Theta$. The prior incorporates known information such as expert opinion or practical experience assumed to be true. The posterior distribution is updated with some experimental data $\mathcal{D}$ by means of the Bayes' rule:

$$
p(\boldsymbol{\Theta} \mid \mathcal{D}, \mathcal{M})=\frac{p(\mathcal{D} \mid \boldsymbol{\Theta}, \mathcal{M}) p(\boldsymbol{\Theta} \mid \mathcal{M})}{p(\mathcal{D} \mid \mathcal{M})}
$$

where $p(\mathcal{D} \mid \Theta, \mathcal{M})$ is the likelihood to obtain the data $\mathcal{D}$ when the value of the updating parameters $\Theta$ is fixed, and a specific model $\mathcal{M}$ is chosen, and the denominator, known as marginal likelihood or evidence, is a normalizing factor that ensures that the posterior distribution integrates to one.

For the updating of finite element in a modal analysis, the data $\mathcal{D}$ is usually the set of eingenvalue residuals $\epsilon_{i}=z_{i}^{e}-z_{i}^{m}(\boldsymbol{\Theta}), i=1 \cdots n$, where $z_{i}^{e}=\omega_{i}^{2}$ is the square of the $i$-th measured natural frequency and $z_{i}^{m}(\boldsymbol{\Theta})=\lambda_{i}(\boldsymbol{\Theta})$ is the $i$-th eigenvalue of a finite element model. In the parameter updating of the modal analysis, the likelihood function is often chosen to be a multivariate normal distribution,

$$
p(\mathcal{D} \mid \boldsymbol{\Theta}, \mathcal{M})=\prod_{i=1}^{N} \frac{1}{(2 \pi)^{n / 2}|\Sigma|^{1 / 2}} \exp \left(-\frac{1}{2} \epsilon_{i}^{T}(\boldsymbol{\Theta}) \boldsymbol{\Sigma}^{-1} \epsilon_{i}(\boldsymbol{\Theta})\right)
$$

where $N$ denotes the number of data points, and the data $\epsilon$ is assumed to be zero-mean and covariance $\boldsymbol{\Sigma}$. The normal likelihood assumption is justified on the basis that the information entropy for a given mean and covariance is maximized by a multivariate normal distribution. In addition, it is customarily assumed in Bayesian model updating that the data are independent and the likelihood is thus

$$
p(\mathcal{D} \mid \Theta, \mathcal{M})=\prod_{i=1}^{N} \frac{1}{\sqrt{2 \pi} \sigma_{i}} \exp \left(-\frac{\epsilon_{i}^{2}}{2 \sigma_{i}^{2}}\right)
$$

It should be noted that is extremely impractical to solve 1 because it requires a solution of a complex and often defined integral, and it is even more involved when multidegree of freedom structural dynamics problems are analysed. Thus, sampling techniques using modern variants of the Markov chain Monte Carlo (MCMC) method are the accepted procedure. In a first step, Metropolis-Hastings (MH) sampling algorithms might be employed to generating samples from a modified posterior without the necessity to solve the highly complex normalization integral, but only by evaluating the product of prior distribution function and likelihood in the $\mathrm{MH}$ acceptance/rejection criterion. It can be shown that convergence is asymptotic upon an equilibrium distribution of the updating parameters with increasing numbers of samples, and if the data is large and sufficiently informative, then convergence is found to be independent of the prior distribution. However, it is generally necessary to discard a considerable number of initial samples in the initial burn-in period to ensure convergence towards the target distribution.

The classical $\mathrm{MH}$ is not suitable for high dimensional problems where the posterior is concentrated in a small region of the sample space so that too many samples are rejected and huge computing resource is required. This restriction has now been overcome to a considerable extent by the development of modern sampling routines efficient variants of the $\mathrm{MH}$ algorithm. Beck and $\mathrm{Au}$ [11] introduced the adaptive MetropolisHastings (AMH) method capable of simulating peaked, flat and multi-modal posterior PDFs. A similar approach using intermediate (or transitional) PDFs was followed by Ching and Chen [12] with the transitional Markov chain MonteCarlo (TMCMC) method overcoming inefficiencies inherent to the use kernel density estimation in AMH by a resampling approach. The method introduces a series of intermediate PDFs, 


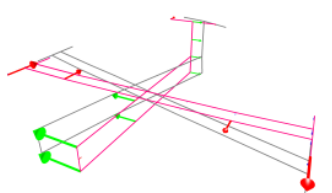

Mode 1: RBM Yaw

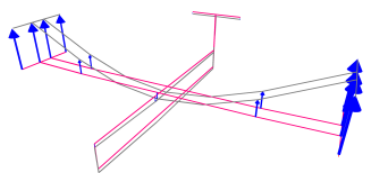

Mode 5: 2n Wing Bending

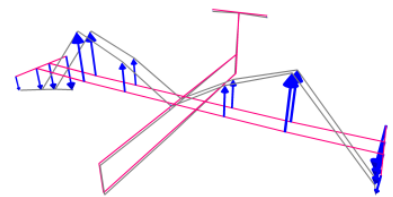

Mode 10: 4n Wing Bending

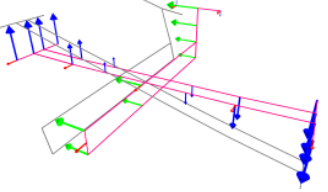

Mode 2: RBM Roll

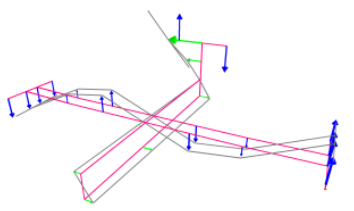

Mode 6: 3n Wing Bending

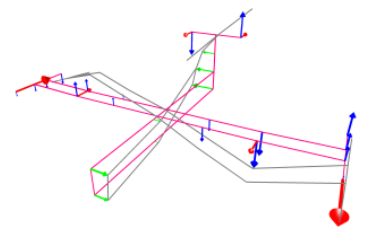

Mode 11: 1n Wing ForeAft

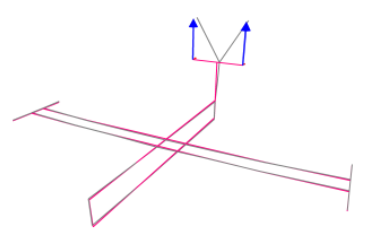

Mode 19: HTP Bending

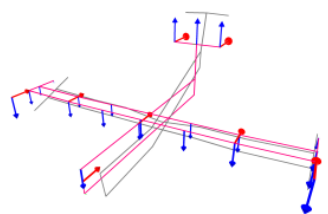

Mode 3: RBM Pitch

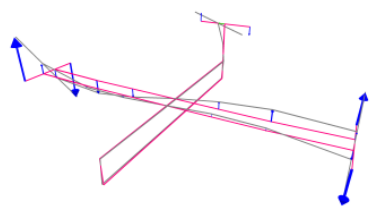

Mode 7: Anti-symm Wing Torsion

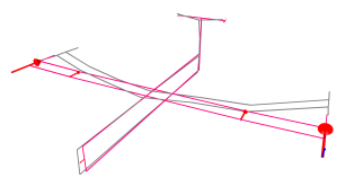

Mode 12: 2n Wing ForeAft

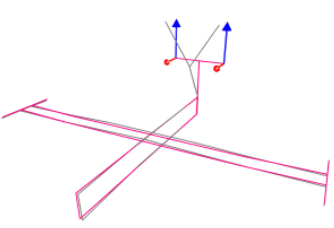

Mode 20: HTP

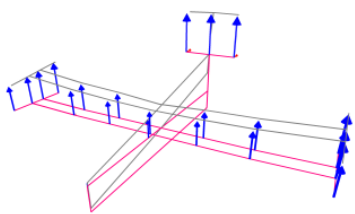

Mode 4: RBM Heave

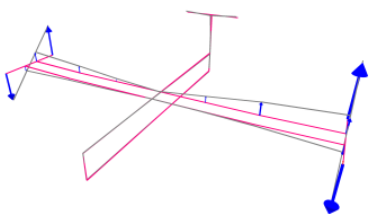

Mode 8: Symm Wing

Torsion

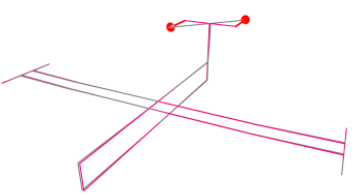

Mode 14: VTP Torsion

Fore-Aft

Fig. 1. Mode shapes used in model updating

$$
\begin{gathered}
p_{k}(\boldsymbol{\Theta})=p(\mathcal{D} \mid \Theta, \mathcal{M})^{\beta_{k}} p(\Theta \mid \mathcal{M}) \\
k=0, \cdots, \mathcal{M} ; 0=\beta_{0}<\beta_{1}<\cdots<\beta_{k}<\cdots<\beta_{M}=1
\end{gathered}
$$

where $k$ denotes the step number of the intermediate posteriors. The series of intermediate distribution starts with the prior distribution and ends with the posterior, thus generating samples from the updated model. In addition, convergence is surely achieved and more effectively than traditional $\mathrm{MH}$ since no burn-in is applied in the sampling scheme for the intermediate distributions. However, many intermediate steps might be required especially if the significant input space of the prior distribution differs greatly from the posterior. In this cases, simulation time can be efficiently improved by employing meta-model while still maintaining the improved convergence characteristics of the TMCMC algorithm.

\section{Multivariate interval identification}

This section explains the interval finite element method, as well as the method that is used for the identification and quantification of multivariate interval uncertainty based on indirect measurement data. In the following, a model parameter $\theta$ having interval uncertainty is denoted $\theta^{I}$. Vectors are expressed as lower-case boldface characters $\boldsymbol{\theta}$. For the remainder of the text, interval parameters are either represented using the bounds of the interval $\theta^{I}=[\underline{\theta} ; \bar{\theta}]$ or the centre point $\hat{\theta}=\frac{\underline{\underline{\theta}+\bar{\theta}}}{2}$ and interval radius $r_{x}=\frac{\bar{\theta}-\underline{\underline{\theta}}}{2}$.

\section{A. The interval finite element method}

Suppose that a finite element (FE) model $\mathcal{M}$ is considered to approximate the solution $z^{m} \in \mathbb{R}^{d}$ of a (set of) differential equations, parametrised by a vector $\boldsymbol{\theta} \in \mathcal{F} \subset \mathbb{R}^{k}$, with $\mathcal{F}$ the subdomain of feasible parameters (e.g., non-negative contact stiffness). In case the elements in the parameter vector $\boldsymbol{\theta}$ are uncertain, $\boldsymbol{\theta}$ is modelled as an interval vector $\boldsymbol{\theta}^{I} \in \mathcal{F}^{I} \subset \mathbb{R}^{k}$ when the interval paradigm is applied. Herein, $\mathbb{I}^{k}$ is the vector space of $k$-dimensional interval vectors. As such, all parameters $\theta$ of the model are considered independent by definition. Then, the interval FE method comes down to finding the solution set $\tilde{\boldsymbol{z}}^{m}$, which usually spans a general nonconvex region in $\mathbb{R}^{d}$. This its computation is in general not 
feasible within polynomial time, it is commonly approximated by an uncertain realization set $\tilde{\boldsymbol{z}}^{m}$, which is obtained by propagating $q$ deterministic realizations $\boldsymbol{z}_{i}^{m}$ of the interval field $\boldsymbol{\theta}^{I}$ :

$$
\tilde{\boldsymbol{z}}^{m}=\left\{\boldsymbol{z}_{i}^{m} \mid \boldsymbol{z}_{i}^{m}=\mathcal{M}\left(\boldsymbol{\theta}_{i}\right) ; \boldsymbol{\theta}_{i} \in \boldsymbol{\theta}^{I} ; i=1, \ldots, q\right\}
$$

These $q$ deterministic propagations should represent the solution set $\tilde{\boldsymbol{z}}^{m}$ as close as possible. In the case of strict monotonicity of $\mathcal{M}()$ the transformation method [13] can be used in this context.

\section{B. Multivariate interval identification}

When a truthfull estimation of $\tilde{\boldsymbol{z}}^{m}$ is needed, the interval uncertainty in $\boldsymbol{\theta}^{I}$ has to be quantified accurately. In case when a direct measurement of the variables is hard to obtain (e.g., when connection stiffness is considered), this can be performed by using indirect measurements of the system responses and a suitable inverse methodology. A generic method was in this context recently introduced by some of the authors [5], [14].

Hereto, a physical representation of the considered model is tested $t$ times in close correspondence with the numerical model, in order to construct a measurement set $\tilde{\boldsymbol{z}}^{e}$. Then, the non-determinism that is present in these replicated measurements is bounded by its convex hull $\mathcal{C}_{e}$. Similarly, also the convex hull $\mathcal{C}_{m}$ of the uncertain realisation set $\tilde{\boldsymbol{z}}^{m}$ is constructed. Moreover, the corresponding $d$-dimensional volumes are computed, as they provide a measure for the enclosed uncertainty. In fact, they can be regarded as an extension of the 1-dimensional interval width to general $d$-dimensions. These computations are performed using the QuickHull algorithm. However, since the time complexity of this algorithm is:

$$
\mathcal{O}\left(\left\lfloor v_{c}^{\frac{d}{2}}\right\rfloor /\left\lfloor\frac{d}{2}\right\rfloor !\right)
$$

with $v_{c}$ the number of vertices of $\mathcal{C}_{m}$ [15], care should be taken when large-dimensional convex hulls need to be constructed. Therefore, the dimension of the vector space $\mathbb{R}^{d}$ in which these convex hull are computed, is reduced using the method presented in [14]. Hereto, an orthogonal basis $\mathcal{B}$ is constructed in $\mathbb{R}^{d_{r}}$, with $d_{r}<<d$, which is defined as:

$$
\mathcal{B}=\left\{\boldsymbol{\phi}_{e, d-d_{r}}, \boldsymbol{\phi}_{e, d-d_{r}+1}, \ldots \boldsymbol{\phi}_{e, d}\right\}
$$

with $d_{r}$ chosen as such that all non-zero dimensions are included in $\mathcal{B}$, and $\phi_{e}$ the eigenvectors corresponding to the $d_{r}$ largest eigenvalues of the covariance matrix of the measurement data set $\tilde{\boldsymbol{z}}^{e}$. The number of needed eigenvectors $d_{r}$ is selected as:

$$
\sum_{i=1}^{d_{r}} \frac{\lambda_{e, i}}{\operatorname{tr}\left(\boldsymbol{\Xi}_{e}\right)} \geq 1-\epsilon
$$

with $\lambda_{e, i}$ the ordered eigenvalues of the covariance matrix $\boldsymbol{\Xi}_{e}$ of the measurement data set, and $\epsilon$ the reduction error. Subsequently, $d_{r}^{+}$-dimensional sub-bases $\mathcal{B}_{i}^{+}$are constructed from
$\mathcal{B}$. Specifically, each possible combination of $d_{r}^{+}$eigenvectors are hereto combined, yielding a total of $\left(\begin{array}{c}d_{r} \\ d_{r}^{+}\end{array}\right)$projections. As such, the computational complexity of determining the bounds on the uncertainty in the model responses becomes:

$$
\mathcal{O}\left(\left\lfloor v_{c}^{\frac{d_{r}^{+}}{2}}\right\rfloor /\left\lfloor\frac{d_{r}^{+}}{2}\right\rfloor ! \times\left(\begin{array}{c}
d_{r} \\
d_{r}^{+}
\end{array}\right)\right)
$$

which is a reduction of computational complexity as long as $d_{r}^{+}$is sufficiently small. Then, the result of the interval FE computation and the measurement data set are projected onto each of these bases $\mathcal{B}_{i}^{+}$and the convex hulls $\mathcal{C}_{e}$ and $\mathcal{C}_{m}$ with their respective volumes is computed in this lower-dimensional basis. Incorporating this in the optimisation problem, presented in eq. (12), this yields:

$$
\delta\left(\boldsymbol{\theta}^{I}\right)=\sum_{i=1}^{\substack{d_{r} \\ d_{r}^{+} \\ d_{i}}}\left(\Delta V_{e}^{2}\left(\mathcal{B}_{i}^{+}\right)+\omega_{0} \times \Delta V_{o}^{2}\left(\mathcal{B}_{i}^{+}\right)+\Delta c^{2}\left(\mathcal{B}_{i}^{+}\right)\right)
$$

with:

$$
\begin{aligned}
& \Delta V_{e}=1-\frac{V_{m}\left(\boldsymbol{\theta}^{I}, \mathcal{B}_{i}^{+}\right)}{V_{e}\left(\mathcal{B}_{i}^{+}\right)} \\
& \Delta V_{o}=1-\frac{V_{o}\left(\boldsymbol{\theta}^{I}, \mathcal{B}_{i}^{+}\right)}{V_{e}\left(\mathcal{B}_{i}^{+}\right)} \\
& \Delta c=\left\|\boldsymbol{c}_{e}-\boldsymbol{c}_{m}\left(\boldsymbol{\theta}^{I}\right)\right\|_{2}
\end{aligned}
$$

and where $\left(\mathcal{B}_{i}^{+}\right)$is used to indicate that the respective multidimensional volumes are computed using the $i^{\text {th }}$ projection of the $d_{r}$ dimensional orthogonal basis. The total error is computed as the sum of squared errors over all considered projections. Furthermore, the parameters $\boldsymbol{c}_{e}$ and $\boldsymbol{c}_{m}$ are the geometrical centres of mass of respectively $\tilde{\boldsymbol{z}}^{e}$ and $\tilde{\boldsymbol{z}}^{m} . V_{o}$ is the multidimensional volume of the overlap $\tilde{\boldsymbol{z}}_{o}$ between $\tilde{\boldsymbol{z}}^{e}$ and $\tilde{\boldsymbol{z}}^{m}$. The weight $\omega_{o}$ can be used to tune the objective function to prioritise maximising the overlap between $\mathcal{C}_{e}$ and $\mathcal{C}_{m}$ or match the volumes of these respective convex hulls. When $\omega_{o}$ is set to a large value (say $1 \cdot 10^{06}$ ), the term $\Delta V_{o}$ acts as a barrier function, forcing $\mathcal{C}_{m} \supseteq \mathcal{C}_{e}$. The interval vector $\boldsymbol{\theta}^{I, *}$ if input parameters is finally determined as :

$$
\begin{gathered}
\boldsymbol{\theta}^{I, *}=\underset{\operatorname{argmin}}{\operatorname{argm}}\left(\delta\left(\boldsymbol{\theta}^{I}\right)\right) \\
\text { s.t. } \boldsymbol{\theta}^{I} \in \mathcal{F}^{I}
\end{gathered}
$$

Since the optimisation problem, introduced in eq. (10) is high dimensional and generally not convex, the Particle Swarm Algorithm was applied prior to calling fmincon, as to ensure that the solver already converged to the vicinity of the global minimum of eq. (10). Specifically, a swarm size of 100 particles with a convergence criterion of 10 stalling generations was found to be sufficient for this problem. From this estimate of the global minimum, fmincon iterates on the objective function until converged (i.e. until the KKT conditions of the optimiser are satisfied [16]). 


\section{NEURAL NETWORK META MODELLING}

For both the interval and the Bayesian method, a considerable amount of deterministic model evaluations are needed. Since each deterministic model evaluation takes approximately 10 seconds of wall-clock time on a Intel Xeon E5-1620 @ 3.70 $\mathrm{GHz}$, performing both methods would take prohibitively long. Therefore, it is proposed to use an artificial neural network metamodel (ANN) for the prediction of the deterministic responses of the FE model. Specifically a 2-layer (16:16:14:1) Neural Network that maps each vector of uncertain model parameter to one eigenfrequency of the FE model is constructed. This specific network lay-out is selected iteratively. Bayesian reguralisation is applied to prevent over-training of the network.

For the training of the ANN, deterministic samples are generated by the FE model of the AIRMOD from a search space covering 0.1 and 10 times the deterministic initial estimate of the model. Since the range of parameters over which the ANN has to provide the model's responses is comparably large, and the location of the global optimum within this range is unknown a priori, the challenge in training the ANN lies in ensuring that the ANN also performs accurately on a tighter interval around the exact global optimum. Therefore, the training data set for the ANN is constructed by propagating 10.000 Latin Hypercube samples of the FE model parameters, drawn from the search space. As such, a dense sampling of the search space is obtained. Such a dense sampling is chosen because the risk exists that adaptive training methodologies yields an ANN that performs well over the entire sampled domain, but not on smaller subdomains.

The generation of the dataset took approximately 24 hours of wall-clock time using only a single core. As concerns the training of the ANN, on average $90 \mathrm{~s}$ are needed to train a single neural network using Bayesian regularisation. As compared to solving the problem using the FE model, this leads to a drastic reduction in wall-clock time, as the generation of the samples and training of the ANN takes less time as compared to solving one interval FE model with the deterministic FE model.

The performance of the ANN is tested by performing another 1000 deterministic samples on the deterministic FE model that are disjunct with the training data. The correlation $R$ between the prediction of the ANN and the FE model is computed for for each considered eigenfrequency, which is on average 0.99989 .

\section{COMPARISON OF BOTH METHODS}

\section{A. Interval identification dimension reduction}

The application of the multivariate interval identification methods needs a reduction of the dimension of the uncertain realisation set prior to computing the convex hulls as a direct result of the complexity of the applied QuickHull algorithm (see eq. (6)). If all 14 selected eigenmodes, as visualised in fig. 1, would be used for the identification, the computation of a 14-dimensional convex hull is needed, which is computationally intractable. Therefore, the dimension of this measurement data set is reduced following the procedure elaborated in section IV-B. In this context, application of equation (8) yields that the original 30-dimensional dataset (i.e., without the prior selection of the 14 eigenmodes) can accurately be represented in a $d_{r}=13$-dimensional orthogonal basis, as the corresponding reduction error $\epsilon$ is $1 \cdot 10^{-03}$. This also can be seen in figure 2, which plots $\epsilon$ as a function of the reduced dimension $d_{r}$. As can be seen, the gain in accuracy in terms of modelled uncertainty when including more than 13 responses is negligible, while the computational expense increases drastically. However the gain from 14 seems negligible at a first glance, it needs to be stressed that the computation of the convex hull has an exponential time complexity with respect to the dimensionality of the problem (eq. (6)). Therefore, based on the singular value decomposition of the covariance matrix of the measured eigenfrequencies of the pre-selected 14 eigenmodes, an orthogonal basis $\mathcal{B} \in \mathbb{R}^{13}$ is constructed according to eq. (7). Then, the measurement data set $\tilde{\boldsymbol{z}}^{e}$ is projected onto this basis.

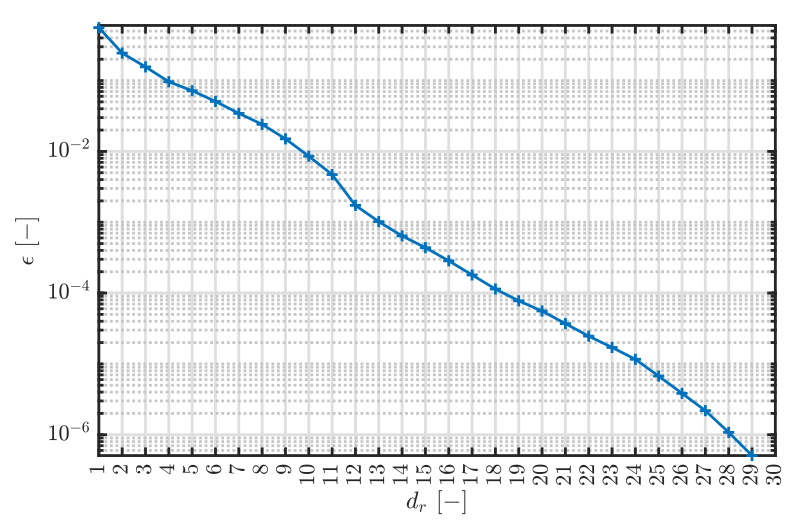

Fig. 2. Reduction error $\epsilon$ as a function of the reduced dimension $d_{r}$, applied to the AIRMOD data set.

However, as the computation of a single 13-dimensional convex hull would take prohibitively long when used in an iterative identification procedure, this dataset is further projected onto lower-dimensional sub-bases $\mathcal{B}_{i}^{+}$, as elaborated in section IV-B. In this context, the dimension of these sub-bases $d_{r}^{+}$is set to 2 , as it this entails the largest gain in computation time. The latter can be seen from equation 9, where it is clear that a low $d_{r}^{+}$also yields the lowest time complexity. Then, based on these lower-dimensional sub-bases, the objective function that is shown in eq. (10) can be minimized to quantify the multivariate interval uncertainty.

\section{B. Results of the AIRMOD identification}

Three aspects of the application of both inverse methods for uncertainty quantification is discussed in this section. First, a general conceptual comparison is provided. Then, the computational cost and conservatism of both methods is critically compared. 


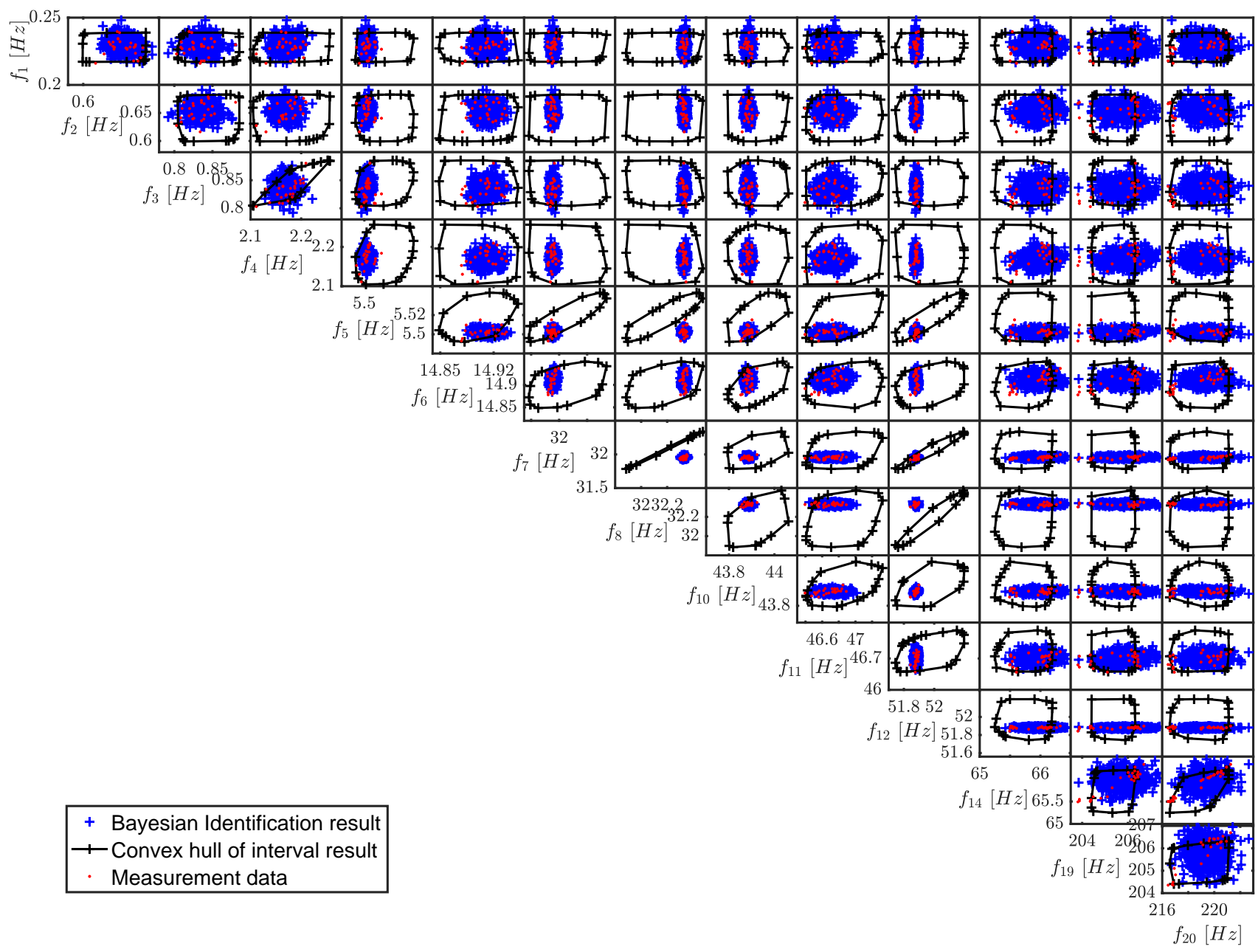

Fig. 3. All measured eigenfrequencies of the AIRMOD structure (as red dots) vs the bounds on the frequencies that are predicted by the iterval FE model with the identified input intervals and 500 realisations of the eigenfrequencies obtained by sampling the posterior of the Bayesian identification

1) Conceptual comparison: Conceptually, the main difference of the Bayesian identification with respect to the interval method, is that the former approaches the problem from inside. By applying the Bayesian identification method, a degree of plausibility for each model response is obtained by sampling the posterior distribution of the uncertain model parameters. As such, when this identification is accurate, also a measure of the system reliability can be obtained. Interval methods on the other hand approach the system from the outside, and aim to encompass all possible responses of the model, given bounds on the uncertain parameters. As such, they do not make any inference on the relative likelihood of the occurrence of each possible response value. Therefore, these results should be interpreted in a worst-case sense. Evidently, when sufficient data are available, as to ensure that an accurate prior can be constructed, Bayesian methods should be applied. However, when only insufficient or vague data are available, interval methods have the upper hand, since they are in this case more objective as no assumptions on the underlying probabilistic nature of the data are needed.

2) Computational aspects: For the Bayesian inference, uniform prior distribution are used for the input parameters, to indicate virtually no knowledge of the AIRMOD physics. The uniform distribution range is of $5 \%-200 \%$ of the nominal parameter values. Finally, the likelihood is defined as in equation 3. 500 Markov Chain samples were used in the TMCMC algorithm, and convergence to the posterior was reached after 21 steps. For each iteration, the Markov chains had an average length of 10 steps to move the sample from the one intermediate distribution to the next, thus a total of approximately $10^{5}$ model evaluation were necessary. By employing both neural networks and thread parallelism for each Markow chain, the simulation took approximately 230 minutes with 12 threads running on a workstation having Intel 
Xeon E5-xxx using CentOS 6 in combination with $64 \mathrm{~Gb}$ of RAM. As such, the Bayesian method is more efficient in this context. It should be noted however that $12 \mathrm{CPU}$ threads where used in this context, as compared to a single thread for the interval identification.

For the interval identification method, the global optimum of eq. (10) was found after 81 generations of the PSO algorithm and another 33 iterations by fmincon, resulting in 9320 interval function evaluations. This corresponds to approximately 420 minutes of wall-clock time on a desktop computer having an Intel Xeon E5-1620 @ 3.70 GHz processor using only a single thread of the CPU using XUbuntu 16.04 in combination with $32 \mathrm{~Gb}$ of RAM. It should be noted that the PSO algorithm is theoretically embarrassingly parallel, and that also a large computational gain in the determination of the gradients of eq. (10) in fmincon can be achieved do to the large number of uncertain parameters. By using the ANN, the combined effort of the training of the network and identification of the uncertainty still proves to be less computationally intensive as compared to propagating the interval uncertainty one time using the deterministic FE model, as one solution of the interval FE model would need 65536 deterministic FE function evaluations.

3) Discussion of the results: Figure 3 shows all combinations of selected eigenfrequencies of the AIRMOD model. The replica from the measurement data set are indicated as red dots, whereas the black lines indicate the convex hull of the interval FE model response, obtained by propagating the interval uncertain model parameters. The blue crosses are 500 realisations of the eigenfrequencies of the AIRMOD, obtained by sampling the identified posterior distributions. For the application of the inverse interval method, it was assumed that the masses at both wing tips are equal. This assumption was not made for the Bayesian inference.

As can be noted, the identified interval model is capable of accurately predicting the spread in the measured eigenfrequencies that were incorporated into the analysis. It may be noted that some values (e.g., considering the $14^{\text {th }}$ or $19^{\text {th }}$ eigenmode) lie outside the predicted intervals, whereas other estimates are too conservative (e.g., the $8^{\text {th }}$ or $10^{\text {th }}$ eigenmode). This is apparent contradiction is caused by the fact that the objective function both aims at minimising the mismatch in volume between the two convex hulls, as on maximising the overlap between these convex hulls. However, the multidimensional volumes cannot be matched perfectly, since the set of measurement data does generally not contain all necessary extreme responses, which biases the multidimensional volume of the measurement data set with respect to the multidimensional volume of the set of interval responses. As such, some sort of equilibrium is found by the optimisers, where the volume is matched at the price of losing some overlap. Moreover, it can also be noted that when the combinations of the $7^{t h}-8^{t h}$ and $8^{t h}-12^{t h}$ eigenfrequency are considered, the convex hull fails to encompass these results, whereas the separate intervals on the parameter responses do provide an accurate prediction of the uncertainty in the model response, albeit somehow conservative. Since the $7^{t h}, 8^{\text {th }}$ and $12^{\text {th }}$ eigenmode correspond to respectively anti-symmetric torsion, symmetric torsion and wing fore-after bending modes, the lack of accurately predicting the dependency between these eigenfrequencies is most probably caused by the assumption that was used in the interval identification that the masses at both wing tips are equal.

From the results of the Bayesian inference, presented in table I, it is also clear that the 500 samples that are obtained by the Bayesian procedure provide a better match with the set of measured replica. However, opposed to the interval method, no finite bounds are given by the Bayesian method, but rather a degree of plausibility for each response value. As such, more samples are located in a region that corresponds to the model parameters that were deemed the most plausible by the Bayesian method. The over-conservatism of interval methods compared to probabilistic methods in inverse UQ was also noted by Govers et al. [4]. A possible explanation for this phenomenon lies in the conceptual differences of both methods, as elaborated earlier on. As a final remark, that a dataset containing 86 specimens can be regarded as a large dataset. It is well-known that Bayesian methods perform very well in this context. Therefore, it could be interesting to evaluate the performance of both methods when only a small subset of this dataset is used for the identification and quantification.

Table I shows the results of the interval identification and Bayesian inference methods using the ANN surrogate model on the AIRMOD data set. The results are scaled by the deterministic initial estimate on the parameter values.

TABLE I

RESULTING PARAMETERS AFTER IDENTIFICATION

\begin{tabular}{lllll}
\hline & \multicolumn{2}{c}{ Interval UQ } & \multicolumn{2}{c}{ Bayes UQ } \\
Param. & $\underline{\theta}_{i}$ & $\bar{\theta}_{i}$ & $\operatorname{mean}(\theta)$ & $\operatorname{std}(\theta)$ \\
\hline$\theta_{1}$ & 0.94 & 1.19 & 1.31 & 0.35 \\
$\theta_{2}$ & 0.98 & 1.07 & 0.84 & 0.20 \\
$\theta_{3}$ & 1.20 & 1.57 & 1.45 & 0.11 \\
$\theta_{4}$ & 0.55 & 0.68 & 0.68 & 0.33 \\
$\theta_{5}$ & 0.55 & 0.68 & 0.53 & 0.31 \\
$\theta_{6}$ & 0.19 & 0.33 & 0.13 & 0.01 \\
$\theta_{7}$ & 0.54 & 1.39 & 1.49 & 0.78 \\
$\theta_{8}$ & 0.81 & 0.93 & 1.00 & 0.17 \\
$\theta_{9}$ & 1.02 & 1.13 & 1.24 & 0.12 \\
$\theta_{10}$ & 1.02 & 1.13 & 1.16 & 0.11 \\
$\theta_{11}$ & 1.73 & 1.99 & 1.57 & 0.34 \\
$\theta_{12}$ & 0.47 & 0.65 & 0.58 & 0.30 \\
$\theta_{13}$ & 0.91 & 0.98 & 1.39 & 0.75 \\
$\theta_{14}$ & 1.81 & 2.27 & 2.04 & 0.41 \\
$\theta_{15}$ & 0.44 & 0.61 & 0.54 & 0.16 \\
$\theta_{16}$ & 0.42 & 0.50 & 0.34 & 0.08 \\
$\theta_{17}$ & 0.01 & 0.31 & 1.07 & 0.72 \\
$\theta_{18}$ & 0.59 & 0.77 & 8.28 & 3.52 \\
\hline
\end{tabular}

The interval quantification, as already mentioned, provides the analyst with crisp bounds between which the uncertain value of the corresponding parameter is believed to lie. No inference on dependence is made, due to the nature of intervals. The outside approach of the interval method is thus also visualised, as only inference on the extreme responses of the 
structure is made.

As concerns the Bayesian result, the mean value (i.e., the most plausible point) and the standard deviation are given. It should be noted that the identified distributions for $\theta_{1}$ and $\theta_{2}$ are bimodal. The presented central moments should therefore be interpreted with care in this context. Next to these distributions, the Bayesian method also provides a measure of correlation between the model parameters. In this context, it was found that a high correlation (i.e., more than $75 \%$ ) exist between $\theta_{1}$ and $\theta_{2}, \theta_{4}$ and $\theta_{5}, \theta_{9}, \theta_{10}$ and $\theta_{11}$ and finally between $\theta_{14}$ and $\theta_{15}$. As is clear, the Bayesian method approaches the problem from the inside by assigning a plausibility distribution to each parameter value and infers dependence, without making inference on the extreme responses of the structure.

\section{CONCLUSION}

This paper presents a comparison between the probabilistic Bayesian method for uncertainty quantification with a novel method for the identification and quantification of multivariate interval uncertainty, based on the AIRMOD test data set. In order to limit the computational expense of the identification associated with both methods, Artificial Neural Networks are trained using Bayesian regularisation to predict the complicated dynamic behaviour of the AIRMOD structure within limited computational cost. The paper gives an elaborate theoretic overview of both the Bayesian and interval methods, as well as discusses the application of both techniques to the AIRMOD structure. Based on the comparison of the obtained results, following conclusions can be made:

- It is possible to obtain a highly accurate quantification of high-dimensional parameter uncertainty using Artifical Neural Network surrogate models for the prediction fo the dynamic response of the system. This is evidenced by both the high $R^{2}$ values of the ANN model prediction, as well as by the accuracy of the performed uncertainty quantification.

- The results that are obtained by applying the Bayesian method are highly accurate, as evidenced by the tight prediction of the uncertain dynamic behaviour of the structure.

- The results that are obtained by applying the multivariate interval identification method are also very accurate, albeit being more conservative. The assumption of dependency between two parameters of the model led to the convex hull over two model responses not encompassing the measurement data, whereas this was obtained in a hypercubic sense.

Future work will be aimed at comparing the performance of both methods in the context of scarce data. It is expected that when decreasing the size of the dataset, at some point the interval method will become more accurate since no assumptions on the underlying probabilistic nature of the data are needed. It will also be investigated if more efficient interval propagation algorithms are applicable in this context.

\section{ACKNOWLEDGEMENTS}

Matthias Faes would like to acknowledge the financial support of the Flemish Research Foundation (FWO) in the frame of travel grants $\mathrm{K} 218117 \mathrm{~N}$ and $\mathrm{K} 217917 \mathrm{~N}$ for staying at the Leibniz University in Hannover. Matteo Broggi and Matthias Faes contributed equally to this paper.

\section{REFERENCES}

[1] I. Elishakoff, "Possible Limitations of Probabilistic Methods in Engineering," Applied Mechanics Reviews, vol. 53, no. 2, p. 19, 2000.

[2] M. Beer and V. Kreinovich, "Interval or moments: Which carry more information?" Soft Computing, vol. 17, no. 8, pp. 1319-1327, 2013.

[3] G. Muscolino, A. Sofi, and M. Zingales, "One-dimensional heterogeneous solids with uncertain elastic modulus in presence of long-range interactions: Interval versus stochastic analysis," Computers and Structures, vol. 122, pp. 217-229, 2013. [Online]. Available: http://dx.doi.org/10.1016/j.compstruc.2013.03.005

[4] Y. Govers, H. Haddad Khodaparast, M. Link, and J. E. Mottershead, "A comparison of two stochastic model updating methods using the DLR AIRMOD test structure," Mechanical Systems and Signal Processing, vol. 52-53, no. 1, pp. 105-114, jul 2015.

[5] M. Faes, J. Cerneels, D. Vandepitte, and D. Moens, "Identification and quantification of multivariate interval uncertainty in finite element models," Computer Methods in Applied Mechanics and Engineering, vol. 315, pp. 896 - 920, 2017.

[6] Y. Govers, H. Haddad Khodaparast, M. Link, and J. E. Mottershead, Stochastic Model Updating of the DLR AIRMOD Structure. [Online]. Available: http://ascelibrary.org/doi/abs/10.1061/9780784413609.049

[7] Y. Govers and M. Link, Using stochastic experimental modal data for identifying stochastic finite element parameters of the AIRMOD benchmark structure, 2012, pp. 4697-4715.

[8] J. Beck and L. Katafygiotis, "Updating models and their uncertainties. i: Bayesian statistical framework," Journal of Engineering Mechanics, vol. 124(4), pp. 445-461, 1998.

[9] L. Katafygiotis and J. Beck, "Updating models and their uncertainties. ii: model identifiability," Journal of Engineering Mechanics, vol. 124(4), pp. 463-467, 1998

[10] K.-V. Yuen, Bayesian Methods for Structural Dynamics and Civil Engineering. John Wiley and Sons, Singapore, 2010.

[11] J. Beck and S.-K. Au, "Bayesian updating of structural models and reliability using markov chain monte-carlo simulation," ASCE, Journal of Engineering Mechanics, vol. 128(4), pp. 380-391, 2002.

[12] J. Ching and Y.-C. Chen, "Transitional markov chain monte carlo method for bayesian model updating, model class selection and mode averaging," ASCE, Journal of Engineering Mechanics, vol. 133, pp. 816832, 2007.

[13] M. Hanss, "The transformation method for the simulation and analysis of systems with uncertain parameters," Fuzzy Sets and Systems, vol. 130, no. 3, pp. 277-289, sep 2002.

[14] M. Faes and D. Moens, "Identification and quantification of spatial interval uncertainty in numerical models," Computers \& Structures, vol. article in press, 2017.

[15] C. B. Barber, D. P. Dobkin, and H. Huhdanpaa, "The quickhull algorithm for convex hulls," ACM Transactions on Mathematical Software, vol. 22, no. 4, pp. 469-483, 1996.

[16] J. Nocedal and S. J. Wright, Numerical Optimization. Springer Verlag, 1999. 